\title{
Tortuosity variation in a low density binary particulate bed
}

\author{
Ricardo Dias, José A. Teixeira, Manuel Mota*, Alexander Yelshin \\ Centro de Eng. Biológica, IBQF, University of Minho, Campus de Gualtar, 4710-057 Braga, Portugal
}

Received 19 June 2005; received in revised form 26 January 2006; accepted 27 January 2006

\begin{abstract}
The importance of particle size ratio and particle composition in the properties of a mixed bed is well known. Nevertheless, the dependence of the bed channel tortuosity $T$ on the porosity $\varepsilon$ in the form $T=1 / \varepsilon^{n}$, where $n$ is assumed to be a constant, shows that the value of $n$ depends on the properties of the packed bed. For loose packing, experimental data for binary mixtures of glass beads of a size ratio from 1 up to 53.8 was analysed in terms of porosity, tortuosity and permeability. The packing procedure was performed without intensive compacting methods e.g. vibration, etc. Obtained results show that the parameter $n$ is a function of the volume fraction of large particles $x_{D}$ and, for spherical particles, lies in the range 0.4-0.5. The explanation for this variation is (1) a distortion effect on the small particles arrangement occurring near the large particle surface; (2) in the region of minimum porosity, near contact points of large particles, the occurrence of dead zones that are free of small particles. A relationship accounting for this effect is proposed that may be useful for the analysis of transport phenomena in granular bed filters, chromatographic columns, etc.
\end{abstract}

(C) 2006 Elsevier B.V. All rights reserved.

Keywords: Particle mixtures; Binary spheres packing; Size ratio; Porosity; Tortuosity

\section{Introduction}

Mixed beds of particles have a wide application in industry and sciences. Granular beds and, in particular, packing of particles with different sizes display a wide range of values of the porosity $(\varepsilon)$ and pore tortuosity $(T)[1-5]$. Models of the binary particle beds porosity [6-14] and permeability [15-19] versus the volume fraction of the mixture components have been thoroughly described.

The tortuosity is defined as $T=L_{\mathrm{e}} / L$, where $L_{\mathrm{e}}$ is the average flow pathway length and $L$ is the bed thickness. Investigations on $T$ are concentrated on the establishment of a relationship between the overall porosity and tortuosity [20]. For granular packings, the main effort has been focused on the determination of a fixed tortuosity value [14,21,22].

For a mixed bed of particles significantly different in size, to take into account the influence of the porosity on the permeability through the tortuosity is of major importance [16]. Due to the different methods applied for packing preparation, the values of bed porosity lie between "loose" and "dense" packing values, making of key importance to know how tortuosity is related with packing porosity.

\footnotetext{
* Corresponding author. Tel.: +351 253604400

E-mail address: MMota@deb.uminho.pt (M. Mota).
}

Among the proposed relations describing the relationship $T$ versus $\varepsilon$ [23-27], for granular packings, a power law relationship is the most frequently used:

$T=\frac{1}{\varepsilon^{n}}$

where $n$ is a numerical value.

There are many evidences that $n$ depends on the packing properties. For binary mixtures, Klusácek and Schneider [28] admitted that $n$ is not a constant. Assuming that in a porous medium there are $m$ classes of pores and that each class occupies the same portion of the total porosity, Millington and Quirk [29] suggested, for unconsolidated systems, $n$ to be $1 / 3$. Zhang and Bishop [30] and Mota et al. [31] applied $n=0.5$. For loose packed spherical particles mixtures the best fit of $T$ versus $\varepsilon$ was obtained for $n=0.4$ [27,32]. $n=0.4$ gives a good approach for tortuosity (1.47 average value) measured in a spheres packing with porosity between $0.363 \pm 0.030$ [21]. Based on these observations, it may be speculated that the exponent index for granular beds describing the dependence of tortuosity on porosity ranges from 0.4 (loose packing) to 0.5 (dense packing).

The above-mentioned assumption can be confirmed by the data of Currie [1] (for sphere mixtures; sand mixtures; spheres/sand mixture) plotted in Fig. 1 together with the plot 


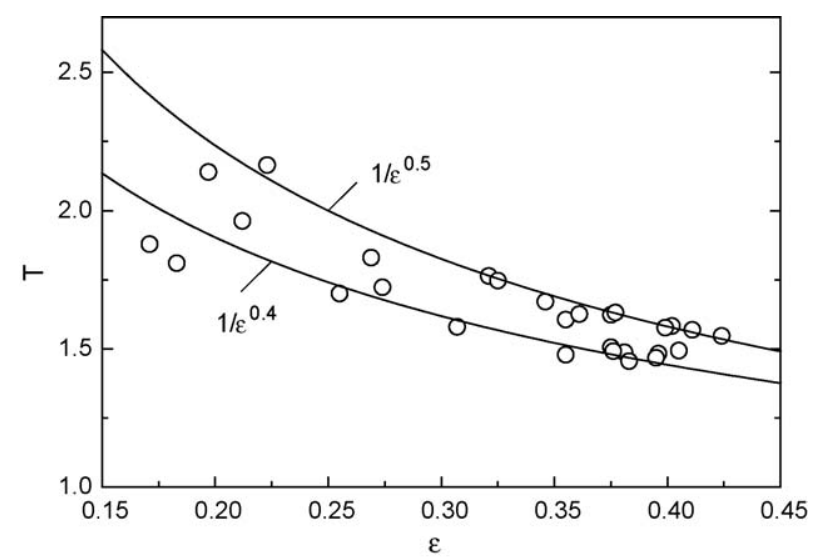

Fig. 1. Dependence of the tortuosity $T$ on the packing porosity $\varepsilon$. Points are plotted using the data from [1] for sphere mixtures, sand mixtures and spheres/sand mixture. Lines represent Eq. (1) for $n$ values of 0.4 and 0.5 .

of Eq. (1) at $n=0.4$ and 0.5 . As can be seen, most of the data lies between the two functions.

Mota et al. [33] investigated a binary mixture spheres at different olume fractions of large particles $x_{D}$ for particle ratios $D / d$ of 13.3, 20, and 26.7. Experiments with the binary particulate bed show that the dependence of $n$ on the fractional content $x_{D}$ lies in the range of 0.5 (for the monosize packing) up to $\sim 0.4$ (in the region of the minimum porosity of the binary bed).

A justification for the variation of the parameter $n$ in Eq. (1) is required with the purpose of establishing a relationship between the binary packed bed porosity, fractional content, tortuosity and, hence, the permeability.

\section{Experimental basis}

Loose packed binary mixtures of glass beads of a size ratio from $D / d=1$ up to 53.8 were analysed in terms of porosity, tortuosity and permeability. Most of the experimental data has been obtained in previous works [27,33] and additional experiments for high $D / d$ ratio were performed using previously described procedures [33-35]. The packing procedure was performed without intensive compacting methods e.g. vibration, etc.

Packing porosity was measured by the volumetric method, whereas the tortuosity and the parameter $n$ in Eq. (1) were determined based on the measurement of permeability.

The permeability $k$ of a mixed bed is characterised by the relation $[3,27]$ :

$$
k=\frac{d_{\mathrm{av}}^{2} \varepsilon^{3}}{\left\{36 K_{0} T^{2}(1-\varepsilon)^{2}\right\}}
$$

where $d_{\mathrm{av}}$ is the average particle size in the bed and for the binary mixture of large particles of size $D$ and small particles of $d$ is defined as $d_{\mathrm{av}}=1 /\left\{x_{D} / D+\left(1-x_{D}\right) / d\right\} ; x_{D}$ is the volume fraction of large particles in the bed; complex $K_{0} T^{2}=K$ is the Kozeny's coefficient and for granular beds $K=4.2 / 5.0 ; T$ is the tortuosity; $K_{0}$ is the shape factor depending on the shape of a capillary pore cross-section area and may be assumed to be $K_{0}=2.0$ for the spheres packing [27,33].
The values of $n$ were calculated from the known porosity, average particle size and permeability using Eq. (3) (obtained from (1) and (2)):

$n=\frac{\ln \left\{36 k K_{0}(1-\varepsilon)^{2} /\left(\varepsilon^{3} d_{\mathrm{av}}^{2}\right)\right\}}{2 \ln (\varepsilon)}$

\section{Analysis and discussion}

Data presented in a previous work [33] shows that the parameter $n$ is a function of $x_{D}$ at the size ratio $D / d=13.3,20$, and 26.7 . Measurements were made for mixtures with $x_{D}$ from 0 up to $x_{D \min }$, this value corresponding to a minimum packing porosity $\varepsilon_{\min }\left(x_{D \min }\right.$ was around $\left.0.65-0.7\right)$. It was shown that the particle arrangement in the binary mixtures at $\varepsilon_{\min }$ is characterised as a loose packing density. As a result, the tortuosity becomes lower being this reflected in the reduction of the parameter $n$ from 0.5 (for monosize packing) up to $\sim 0.4$ (at the minimum binary packing porosity).

The reason for $n$ variation may be explained by the distortion effect of the small particles packing occurring near the large particles surface. In Fig. 2, a sketch of a binary packing at $D / d=20$ is shown, where the distortion effect is represented. Two types of distortion may be considered: when the volume fraction in the mixture, $x_{D}$, is below the minimum porosity region (the skeleton formation in the mixture [34,35]), Fig. 2a, the main distortion occurs near the large particles surface; when $x_{D}$ approaches the region of minimum porosity, where large particles tend to form aggregates, becoming the skeleton at $\sim x_{D \min }$, a small particles jam occurs near the contact points of large particles and dead zones free of small particles are formed, Fig. 2b. Both types of distortion are responsible for $n$ becoming smaller than 0.5.

The volume of the packing involved in the distortion presents a complex dependence on $\delta=d / D[33,35]$ and increases, when $\delta$ is reduced from 1.0, reaches its maximum value at $\delta$ between 0.1 and 0.01 , and diminishes when the loose packing minimum porosity approaches the dense packing values at $\delta<0.01$. At $\delta \rightarrow 0$ loose and dense packing properties are convergent, hence, $n=0.5$ may be expected.

To verify that the packing density obtained in the experiments at $x_{D \min }$ corresponds to a loose packing, comparison with the data presented in works $[27,33]$ as well as with the boundary limits [33] of loose (Eq. (4)) and dense (Eq. (5)) packings at $x_{D} \leq x_{D \min }$ was done.

$$
\begin{aligned}
& \varepsilon_{\min }=\frac{\varepsilon_{d}^{0}\left(1-x_{D \min }\right) \exp \left(1.2264 x_{D \min }^{1 / \sqrt{\delta}}\right)}{1-\varepsilon_{d}^{0} x_{D \min }} \\
& \varepsilon_{\min }=\varepsilon_{\min }+\left(\varepsilon_{d}^{0}-\varepsilon_{\min }\right) \exp \left[0.25\left(1-\frac{1}{\delta}\right)\right]
\end{aligned}
$$

$x_{D \min }$ is the large size particle volume fraction in the mixture when packing has minimum porosity; $\varepsilon_{\min }$ is the absolute minimum porosity of the mixed bed when $\delta \rightarrow 0$ and distortion effect becomes insignificant $\varepsilon_{\min }=\varepsilon_{D}^{0} \varepsilon_{d}^{0}, \varepsilon_{D}^{0}$, and $\varepsilon_{d}^{0}$ are, respectively, the porosity of a pure packing of the large and small size particles. 


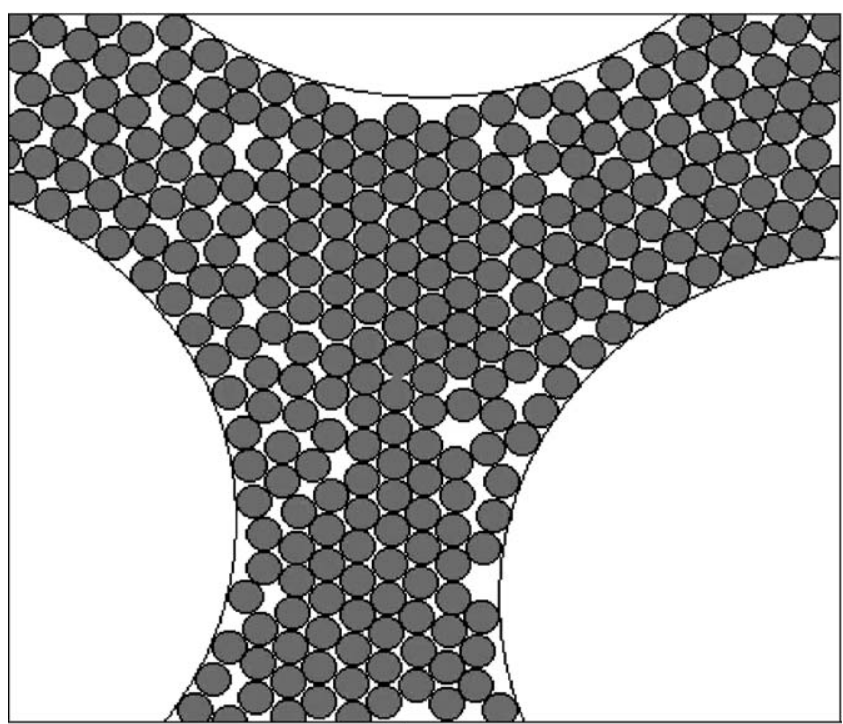

(a)

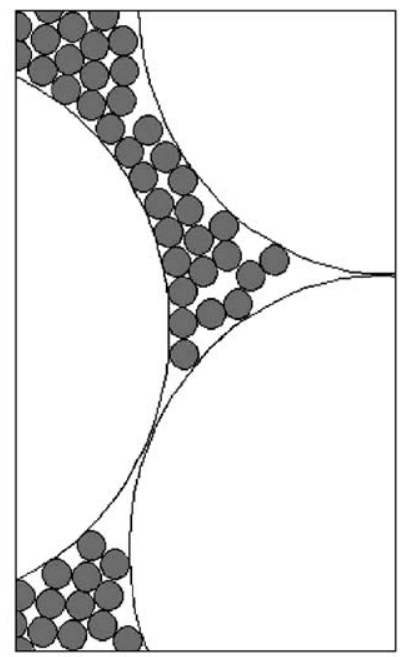

(b)

Fig. 2. Representation of a binary packing at $D / d=20$ showing the distortion effect in the small size particle fraction: (a) $x_{D}$ below the skeleton formation in the mixture; (b) $x_{D}$ in the region of the minimum porosity.

In Fig. 3, experimental values of the minimum porosity are plotted versus particle size ratio $\delta$ together with the boundary values obtained by Eqs. (4) and (5) (curves 1-4). Curves 1 and 2 , represent values obtained at $x_{D \min }=0.71$ for $\varepsilon_{d}^{0}=0.41$ and 0.38, using Eq. (4). Curves 3 and 4, are obtained with Eq. (5), for $\varepsilon_{\min }=0.16$ and 0.1296 , respectively. These values, if $\varepsilon_{D}^{0}=$ $\varepsilon_{d}^{0}$ is assumed, correspond to the monosize packing porosities of $0.4\left(\varepsilon_{\min }=0.16\right)$ and $0.36\left(\varepsilon_{\min }=0.1296\right)$. Curves 1 and 2 outline the loose packing region, whereas curves 3 and 4 show a dense packing area. As can be seen from Fig. 3, all the discussed experimental values belong to the loose packing region.

Using Eq. (3) it is possible to know whether $n$ is a constant or a variable at different fractional contents $x_{D}$. Obtained results are shown in Fig. 4 together with Boltzmann fitting curves (http://www.originlab.com).

It is clearly seen that $n$ is not a constant and lies in range $0.4-0.5$ for the investigated loose packings. The reduction of $n$

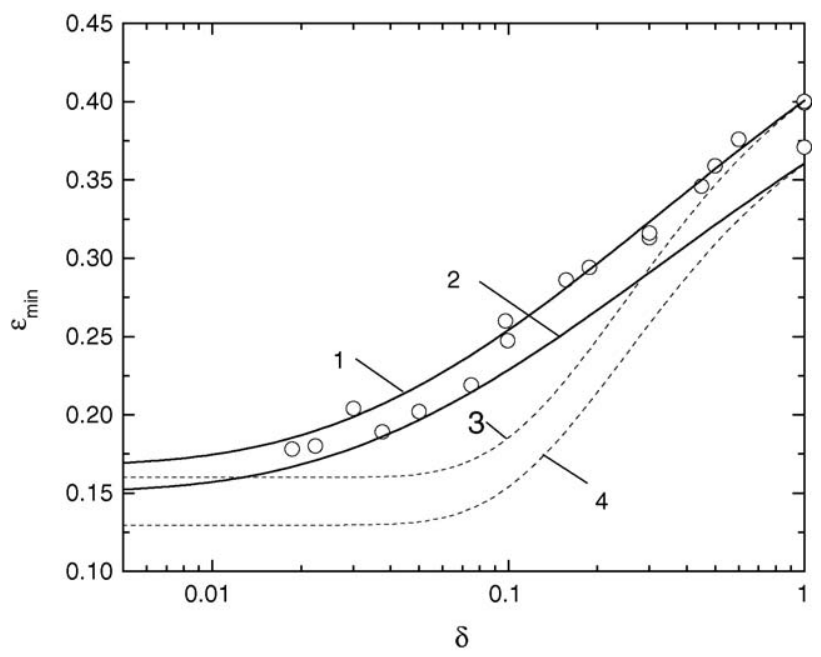

Fig. 3. Dependence the binary packing minimum porosity $\varepsilon_{\min }$ on the ratio $\delta=d / D$ based on previous obtained $[27,33]$ and recent experimental data. Curves 1 and 2, Eq. (4), calculated at $x_{D \min }=0.71$ for $\varepsilon_{d}^{0}=0.41$ and 0.38 . Curves 3 and 4 , Eq. (5), when $\varepsilon_{\min }=0.16$ and 0.1296 , respectively.

when the system approaches minimum porosity can be explained with the small size particle packing distortion in the regions close to the large particles surface and the particles jamming in the void of the large particles skeleton, Fig. 2. From Fig. 3, it is also possible to consider that an increase in particles size ratio diminishes this effect. As no data is available for $D / d$ higher 53.8, a theoretical approach is presented below.

The converging of the loose and dense packing $\varepsilon_{\min }$ may be explained by the reduction in the distortion effects of both types for $\delta \ll 1.0$. This fact is confirmed by the results presented by Bouvard and Lange [36]. These authors simulated a coordinate number in a binary system of spherical particles of different size for two cases: (1) when inclusions touch a matrix particle (coordinate number $Z$ ) and (2) when they touch a plane wall (coordinate number $Z_{\mathrm{w}}$ ). The obtained dependence of $Z_{\mathrm{w}} / Z$ on $\delta$ is shown in Fig. 5, being clearly shown that the coordinate number ratio approaches unity at the same region of $\delta$ as in Fig. 3.

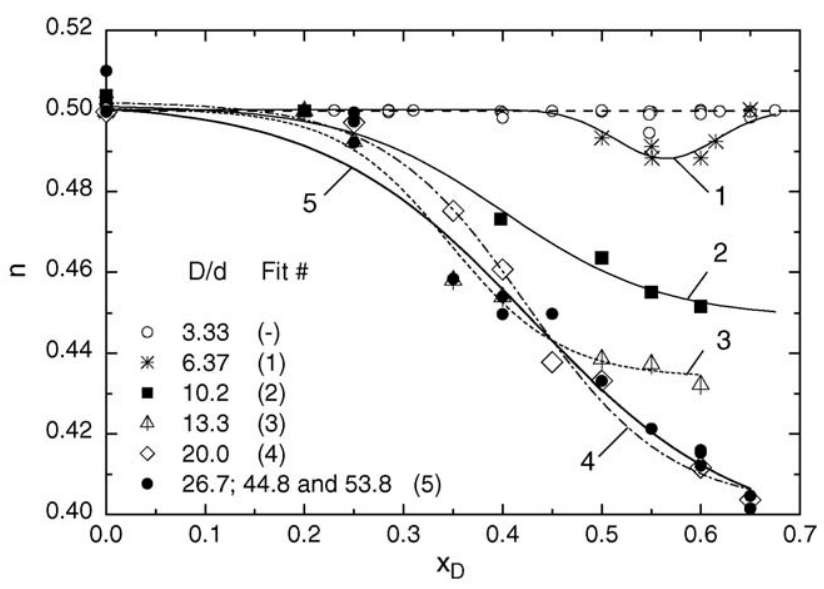

Fig. 4. Dependence $n$ on $x_{D}$ for differents $D / d$. 


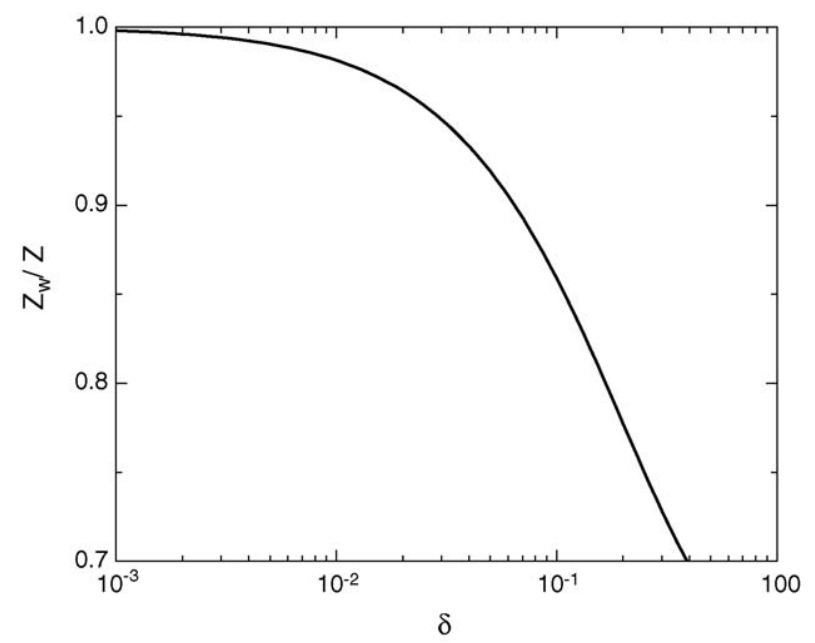

Fig. 5. Dependence of $Z_{\mathrm{w}} / Z$ on $\delta$ according to Bouvard and Lange [36].

In previous works [33,35], different regions corresponding to different types of packing effects could be identified, based in the obtained relation of $\varepsilon_{\min }$ versus $\delta$. According to the mentioned results, regions may be identified as: (1) a region of $\delta>0.1-0.2$ where a displacement mechanism acts upon particles with transition to a linear - mixing model with a decrease of $\delta$ and where displacement and mixing effects in conjunction with packing distortion reach a maximum around $\delta \sim 0.1$; (2) a region of partially disturbed arrangement of small particles in the void of the skeleton, $0.01<\delta<0.1$; (3) a region of small particles arrangement approaching monosize packing, $\delta<0.01$.

As a first step, it is possible to assume that the distortion effects at the loose packing correspond to a statistical "noise" or "defects" distribution acting on the dependence of $n$ versus $\delta$. The simplest representation of this effect would be a Gaussian distribution function $n=f(\delta)$, but due to the non-linear scale of propagation of the different effects along the axis $\delta$ a lognormal distribution function is more adequate (Fig. 6).

The results presented in Fig. 6 confirm the above considered assumptions. Lognormal distribution minima and width

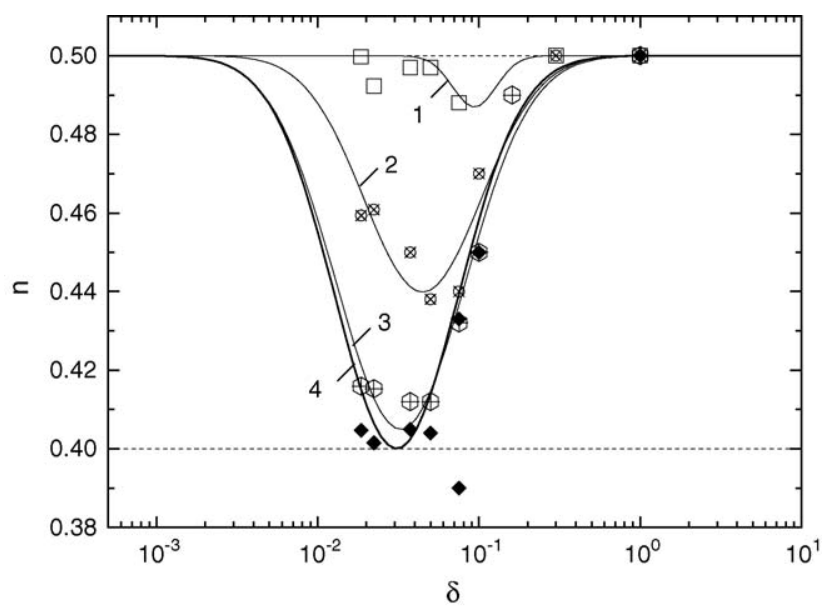

Fig. 6. Fitted lognormal distribution functions of the determined dependence of $n$ on $\delta$ for different $x_{D}: 1-0.25,2-0.45,3-0.6$, and $4-0.65$ (minimum porosity packing region).

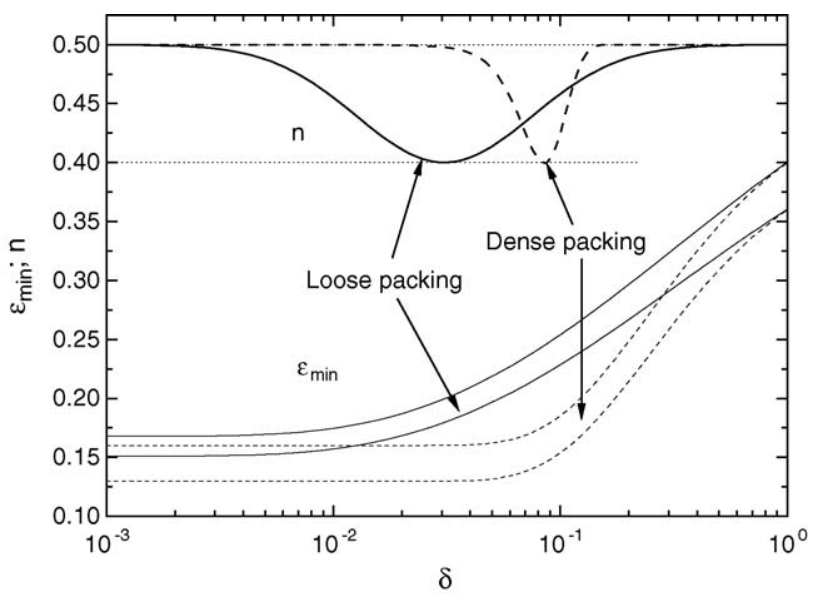

Fig. 7. Dependence of $n$ and $\varepsilon_{\min }$ (from Fig. 3) on $\delta$. Solid and dashed lines represent loose and dense packings, respectively, for $\varepsilon_{\min }$ vs. $\delta$ as well for $n$ vs. $\delta$. Dependence $n$ vs. $\delta$ represents for loose and dense packings by Gaussian and lognormal distribution functions, respectively.

correspond to the region where the maximum distortion effect is observed [33,35]. It is well seen that the coordinate number ratio approaches unity at the same region of $\delta$ as in Fig. 6. The minimum $n$ (maximum distortion) moves to lower values of $\delta$ as the packing goes from monosize packing $x_{D}=0$ to binary packing at $x_{D \max }$ as the contact area between small and large particles reaches its maximum value.

For dense packings, the distortion effect is minimal and it is likely that the "defects" distribution function is of the Gaussian type. Comparison of the dependence of $n$ and $\varepsilon_{\min }$ (from Fig. 3) on $\delta$ for both loose (solid lines) and dense packings (dashed lines) is shown in Fig. 7. For both packings, the dependence of $n$ on $\delta$ is represented by lognormal and Gaussian distribution functions. Lognormal and Gaussian peak positions and width do agree with the regions where the maximum distortion effect is observed.

Let us consider, for a loose packing, a possible error at the region of minimum $n$ ( $\delta$ as 0.03 ) in comparison with the conventionally used $n=0.5$, as well as a similarity between loose and dense packings. For this purpose, Eq. (2) can be converted into the dimensionless form $k / d^{2}=\varepsilon^{3} /\left(K_{0} T^{2}(1-\varepsilon)^{2}\right)$ making it possible to calculate the ratio of $k / d^{2}$ for the two above mentioned cases for the minimum porosity $\varepsilon_{\min }$ and tortuosity (1).

In the first case, for the minimum value of $n=0.4$, the porosity is $\varepsilon_{\min } \sim 0.19$ and, consequently, the error in the calculation is $\left(k / d^{2}\right)_{n=0.5} /\left(k / d^{2}\right)_{n=0.4}=1.394$. This is a significant value for permeability determination.

For the second case, at $\delta=0.03$, for the dense packing $n=0.5$ and $\varepsilon_{\min } \sim 0.145$ can be used. The resulting ratio becomes $\left(k / d^{2}\right)_{\text {Loose }} /\left(k / d^{2}\right)_{\text {Dense }}=4.58$. As a result of the expected differences in $n$ for the loose and dense packings together with the differences in porosity, a significant gap in the packing permeability $\left(k / d^{2}\right)_{\text {Loose }} /\left(k / d^{2}\right)_{\text {Dense }}$ must be observed in the region of $\delta \sim 0.02-0.25$.

Although these estimations need further experimental validation, the obtained data are useful for the improvement of packing density control in different applications. 


\section{Conclusion}

The complexity of the processes involved in the formation of granular beds results in the inter-dependence of the main parameters included in the permeability: packing porosity and tortuosity. The bed porosity in the region of $\varepsilon_{\min }$ is affected by particle size ratio and packing fractional content.

The obtained results show that the parameter $n$ in the tortuosity formula $T=1 / \varepsilon^{n}$ is a function of the packing content $x_{D}$ and lies in the range $0.4-0.5$. The reason for $n$ variation may be explained by the distortion effect of the small particles arrangement occurring near the large particle surface and a jamming effect. It was shown that for the loose packing the most probable deviation from the permeability calculated at $n=0.5$ is expected to be in a wider range of $\delta$ than for the dense packing with a maximum around $\delta=0.03$.

The relationship accounting for this effect may be useful for transport phenomena analysis in granular media.

\section{Acknowledgements}

The authors wish to thank Fundação para a Ciência e Tecnologia (FCT) for having provided the funds to perform this work through the project POCTI/EQU/37500/2001, as well as for the grant accorded to A. Yelshin. This project was partially funded by FEDER.

\section{References}

[1] J.A. Currie, Br. J. Appl. Phys. 11 (1960) 318-324.

[2] C.N. Satterfield, Mass Transfer in Heterogeneous Catalysis, MIT Press, Cambridge, 1970.

[3] J. Bear, Dynamics of Fluids in Porous Media, Elsevier, New York, 1972.

[4] J. Bear, Y. Bachmat, Fundamentals of transport phenomena in porous media, in: J. Bear, M.Y. Corapcioglu (Eds.), NATO ASI Series E: Applied Sciences, vol. 82, Nijhoff Publisher, The Netherlands, 1984, pp. 3-61.

[5] R.H. Perry, D.W. Green, J.O. Maloney (Eds.), Perry's Chemical Engineers Handbook, McGrow-Hill, 1988, pp. 5-54.

[6] N. Ouchiyama, T. Tanaka, Ind. Eng. Chem. Fundam. 20 (1981) 66-71.
[7] A.B. Yu, R.P. Zou, N. Standish, Ind. Eng. Chem. Res. 35 (1996) 3730-3741.

[8] Z.Z. Hulewicz, Int. Chem. Eng. 27 (1987) 566-573.

[9] M. Suzuki, K. Makino, M. Yamada, K. Iinoya, Int. Chem. Eng. 21 (1981) 482-488.

[10] K. Gotoh, T. Chuba, A. Suzuki, Int. Chem. Eng. 22 (1982) 107-115.

[11] M. Kuramae, Int. Chem. Eng. 22 (1982) 666-673.

[12] A.B. Yu, N. Standish, Ind. Eng. Chem. Res. 30 (1991) 1372-1385.

[13] O. Macé, J. Wei, Ind. Eng. Chem. Res. 30 (1991) 909-918.

[14] M.J. MacDonald, C.-F. Chu, P.P. Guilloit, K.M. Ng, AIChE J. 37 (1991) 1583-1588.

[15] R. Jeschar, Arch Eisenhüttenwesen 35 (1964) 91-108.

[16] R. Ben Aim, P. Le Goff, P. Le Lec, Powder Technol. 5 (1971) 51-60.

[17] F.A.L. Dullien, Chem. Eng. J. 10 (1975) 1-34.

[18] R.S. Maier, D.M. Kroll, H.T. Davis, R.S. Bernard, J. Colloid Interface Sci. 217 (1999) 341-347.

[19] D. Wu, L. Song, B. Zhang, Y. Li, Chem. Eng. Sci. 58 (2003) 3995-4004.

[20] A. Revil, J. Colloid Interface Sci. 212 (1999) 503-522.

[21] D.G. Huizenga, D.M. Smith, AIChE J. 32 (1986) 1-6.

[22] T. Wright, D.M. Smith, D.L. Stermer, Ind. Eng. Chem. Res. 26 (1987) $1227-1232$

[23] F.-G. Ho, W. Strieder, Chem. Eng. Sci. 36 (1981) 253-258.

[24] H. Pape, L. Riepe, J.R. Schopper, Colloids Surf. 27 (1987) 97-122.

[25] M.R. Riley, F.J. Muzzio, H.M. Buettner, S.C. Reyes, Biotechnol. Bioeng. 49 (1996) 223-227.

[26] E. Mauret, M. Renaud, Chem. Eng. Sci. 52 (1997) 1807-1817.

[27] M. Mota, J.A. Teixeira, A. Yelshin, Trans. Filtration Soc. 1 (2001) 101106.

[28] K. Klusácek, P. Schneider, Chem. Eng. Sci. 36 (1981) 523-527.

[29] R.J. Millington, J.P. Quirk, Trans. Faraday Soc. 57 (1961) 1200-1207.

[30] T.C. Zhang, P.L. Bishop, Water Res. 28 (1994) 2279-2287.

[31] A. Yelshin, M. Mota, J. Teixeira, Proceedings of the International Conference on Filtech Europa-97, Düsseldorf, Filtration Society, Horsham, UK, October 14-16, 1997, pp. 327-334.

[32] M. Mota, J.A. Teixeira, A. Yelshin, in: Ed. Feyo de Azevedo, E. Ferreira, K. Luben, O Osseweijer (Eds.), Proceedings of the Second European Symposium on Biochemical Engineering Science, Univ. of Porto, Porto, Portugal, September 16-19, 1998, pp. 93-98.

[33] M. Mota, J.A. Teixeira, R. Dias, A. Yelshin, Proceedings of the Ninth World Filtration Congress, New Orleans, Louisiana, AFS, Paper 316-3, April 18-22, 2004, pp. 1-30.

[34] R. Dias, J.A. Teixeira, M. Mota, A. Yelshin, Sep. Purif. Technol. 37 (2004) 69-80.

[35] R. Dias, J.A. Teixeira, M. Mota, A. Yelshin, Ind. Eng. Chem. Res. 43 (2004) 7912-7919.

[36] D. Bouvard, F.F. Lange, Phys. Rev. A 45 (1992) 5690-5693. 